Revta brasil. Bot., São Paulo, V.24, n.3, p.263-272, set. 2001

\title{
Composição, estrutura e relações florísticas do componente arbóreo de uma floresta estacional no Rio Grande do Sul, Brasil
}

\author{
JOÃO ANDRÉ JARENKOW ${ }^{1,2}$ e JORGE LUIZ WAECHTER ${ }^{1}$
}

(recebido: 12 de abril de 2000; aceito: 6 de junho de 2001)

\begin{abstract}
Composition, structure and floristic relations of the tree component of a seasonal forest in Rio Grande do Sul, Brazil). Species composition of seasonal forests in Rio Grande do Sul is strongly influenced by two different floristic contingents, one coinciding with the eastern Atlantic forests and other with the western Paranean-Uruguayan forests. A phytosociological survey of a central South-Riograndean forest was carried out to describe the community structure of the tree component and the local participation of different floristic contingents. All trees with DBH $\geq 5 \mathrm{~cm}$ were recorded in an area of 1 ha, subdivided into 100 square plots of $10 \times 10 \mathrm{~m}$. The sampled species were classified according to their geographic distribution into widespread and restricted to eastern or western floristic affinities. The total density per hectare were 1855 individuals, distributed into 23 families, 46 genera and 55 species. The families with highest species richness were Fabaceae and Myrtaceae. The most important species were Gymnanthes concolor Spreng., Euterpe edulis Mart., Sorocea bonplandii (Baill.) Burger, Lanj. \& Boer, Pachystroma longifolium (Nees) I.M. Johnst. and Trichilia claussenii C.DC., performing 55.2\% of the total of importance values. Species diversity (H') was estimated as 2.244 (nats). The western species form a more diversified contingent, generally occuring as canopy or emergent trees. The eastern species constitute a much less diversified contingent, however with a high quantitative participation as medium-tall trees of the forest understorey. General composition and structural features suggest the importance of canopy trees of interior seasonal forests as providing a suitable habitat for several understorey trees from coastal rain forests.
\end{abstract}

RESUMO - (Composição, estrutura e relações florísticas do componente arbóreo de uma floresta estacional no Rio Grande do Sul, Brasil). A composição específica de florestas estacionais no Rio Grande do Sul é fortemente influenciada por dois contingentes florísticos diferentes, um coincidente com as florestas atlânticas do leste e outro com as florestas paranaenseuruguaias do oeste. Um levantamento fitossociológico de uma floresta central sul-rio-grandense foi realizado para detectar a estrutura comunitária do componente arbóreo e a participação local dos diferentes contingentes florísticos. Todas as árvores com DAP $\geq 5 \mathrm{~cm}$ foram registradas em uma área de 1 ha, subdividida em 100 unidades amostrais de $10 \times 10 \mathrm{~m}$. As espécies amostradas foram classificadas como amplas ou restritas, segundo suas distribuições geográficas, considerando as afinidades florísticas leste ou oeste. A densidade total por hectare foi de 1855 indivíduos, pertencentes a 23 famílias, 46 gêneros e 55 espécies. As famílias com maior riqueza específica foram Fabaceae e Myrtaceae. As espécies mais importantes foram Gymnanthes concolor Spreng., Euterpe edulis Mart., Sorocea bonplandii (Baill.) Burger, Lanj. \& Boer, Pachystroma longifolium (Nees) I.M. Johnst. e Trichilia claussenii C.DC., acumulando 55,2\% do total do valor de importância. A diversidade específica (H') foi estimada em 2,244 (nats). As espécies do oeste formam um contingente mais diversificado, geralmente ocorrendo como árvores do dossel ou emergentes. As espécies do leste constituem um contingente bem menos diversificado, porém com uma alta participação quantitativa como árvores de porte médio do sub-bosque. Aspectos gerais da composição e da estrutura sugerem a importância de árvores do dossel de florestas estacionais interiores como formadoras de ambientes favoráveis para diversas árvores do sub-bosque de florestas pluviais costeiras.

Key words - Floristics, seasonal forest, phytosociology, phytogeography, southern Brazil

\section{Introdução}

As características climáticas predominantes no Rio Grande do Sul configuram uma situação favorável ao desenvolvimento de formações florestais, embora tenha sido estimado que somente cerca de $60 \%$ da cobertura original se constituía de florestas. A maior parte destas formações situava-se na metade norte

1. Universidade Federal do Rio Grande do Sul, Departamento de Botânica, Av. Paulo Gama, 40, 90046-900 Porto Alegre, RS, Brasil.

2. Autor para correspondência: jarenkow@portoweb.com.br do estado, com limite aproximadamente coincidente com o paralelo $30^{\circ} \mathrm{S}$ (Lindman 1906, Rambo 1956).

A geomorfologia, juntamente com o clima, tem um papel destacado, entre os fatores interativos que determinam a concentração de florestas na porção norte do estado. $\mathrm{O}$ aspecto geomorfológico mais importante é a presença de um extenso planalto de constituição predominantemente basáltica, o Planalto Sul-Brasileiro, cujas bordas erodidas são denominadas, impropriamente, de Serra Geral (Rambo 1956, Leite \& Klein 1990).

O Planalto Sul-Brasileiro apresenta uma face dominante voltada para o leste, a encosta atlântica, que no estado vai de Torres até Osório, estendendo- 
se depois para o oeste, com um lento decréscimo altitudinal, indo além de Santa Maria, constituindo a encosta meridional. A vegetação que recobre essas encostas e as depressões adjacentes foi denominada, genericamente, de Mata da Fralda da Serra Geral (Lindman 1906, Rambo 1956, Klein 1983).

Sobre a encosta leste acha-se a extremidade sul da distribuição da Mata Atlântica ou Floresta Ombrófila Densa, na forma de um estreito corredor, entre as porções mais elevadas do planalto, com altitudes médias em torno de $1000 \mathrm{~m}$, e as formações pioneiras litorâneas (Klein 1961, 1984a, Baptista 1976, Veloso \& Góes-Filho 1982, Leite \& Klein 1990).

Na vertente meridional do Planalto Sul-Brasileiro, estende-se uma densa área florestal designada Mata da Encosta Meridional ou das Bacias dos Rios JacuíIbicuí, que na classificação do Projeto RADAMBRASIL apresenta duas porções distintas: a Floresta Estacional Semidecidual, coincidente com a bacia do rio dos Sinos, e a Floresta Estacional Decidual, com área de ocorrência mais ampla, entre os rios Caí e Itu, este último na bacia do rio Ibicuí (Veloso \& Góes-Filho 1982, Teixeira et al. 1986).

A tropicalidade das florestas de encosta, manifestada pela presença de um número relativamente elevado de espécies e gêneros, levou Rambo $(1951,1961)$ a apontar a Mata Atlântica, pelo leste, e a Mata das Bacias dos Rios Paraná-Uruguai, pelo oeste, como os dois principais corredores de imigração de espécies tropicais que se estendem até o Rio Grande do Sul. As condições climáticas do planalto, no sul do Brasil, impedem a transposição de espécies de ambos os contingentes, suscetíveis ao frio, de um lado para o outro.

Nas porções rebaixadas do planalto, na região do Alto Uruguai, um considerável número de espécies características do contingente oeste consegue ultrapassar os divisores de águas e, descendo ao longo de vales de afluentes do rio Jacuí, chegar à encosta meridional e mesmo mais para o sul (Rambo 1951, 1961, Klein 1984a, Veloso \& Góes-Filho 1982). Pelo lado atlântico, as espécies tropicais chegam ao estado pela "Porta de Torres", a maioria distribuindose aproximadamente até a altura de Osório. Algumas, a partir daí, contornam a borda do planalto, dirigindose para o oeste. Outras igualmente conseguem chegar mais ao sul, pelas encostas do Escudo Cristalino SulRio-Grandense e Planície Costeira, formando um gradiente decrescente de riqueza específica (Rambo 1956, 1960, Waechter 1998).
As florestas ocorrentes ao longo das encostas apresentam grande complexidade estrutural, já descrita nos trabalhos pioneiros de Lindman (1906) e Rambo (1956). Abordagens posteriores contribuem com descrições gerais da vegetação ou levantamentos florísticos (Schultz 1957, Baptista 1967, 1976, Klein 1983, 1984b, Reitz et al. 1983) e apresentam também estimativas de parâmetros fitossociológicos (IBDF 1983, Longhi et al. 1986, Machado \& Longhi 1991, Daniel 1991, Farias et al. 1994, Bencke \& Soares 1998).

As encostas do Planalto Sul-Brasileiro, principalmente na face meridional, mostram-se como áreas privilegiadas para o desenvolvimento de estudos ecológicos e fitogeográficos, por se constituírem no local de encontro e interpenetração de dois contingentes tropicais, o oeste, caracteristicamente mesófilo ou estacional (interior) e o leste, higrófilo ou pluvial (atlântico). Para Rambo (1961), o contingente oeste seria mais importante, tanto em riqueza específica quanto em amplitude geográfica, e portanto, geologicamente mais antigo.

$\mathrm{O}$ presente estudo objetiva conhecer a composição florística, a estrutura comunitária e a proporção de espécies pertencentes aos contingentes florísticos do leste, do oeste e o de ampla distribuição, na constituição do componente arbóreo de uma floresta estacional na encosta meridional do Planalto Sul-Brasileiro. Este conhecimento permitirá uma primeira quantificação dos gradientes florísticos existentes ao longo da extensa faixa de florestas da Serra Geral, no Rio Grande do Sul.

\section{Material e métodos}

A área de estudo situa-se no município de Vale do Sol (figura 1), na localidade denominada de Linha XV de Novembro $\left(29^{\circ} 34^{\prime} \mathrm{S}\right.$ e $\left.52^{\circ} 40^{\prime} \mathrm{W}\right)$. A floresta cobre uma encosta com exposição sul, entre altitudes que variam de 100 a $140 \mathrm{~m}$, apresentando-se com fisionomia uniforme e relativamente bem preservada, não aparentando sinais de alterações recentes na vegetação, com exceção do corte de alguns palmiteiros (Euterpe edulis).

As encostas, do ponto de vista geológico, inserem-se na formação Serra Geral, originada de uma intensa manifestação de vulcanismos fissurais no final do Jurássico e no Cretáceo, resultando em vários derrames de lava sobre a Formação Botucatu. Em seu estágio inicial, as lavas são de caráter predominantemente básico, com uma seqüência superior de efusivas ácidas (Kaul 1990). O clima na encosta meridional é subtropical úmido (Cfa) pela classificação de Köppen (Moreno 1961). Os dados coletados pela Estação Meteorológica de Santa Cruz, a mais próxima (cerca de $28 \mathrm{~km}$ em linha reta), 


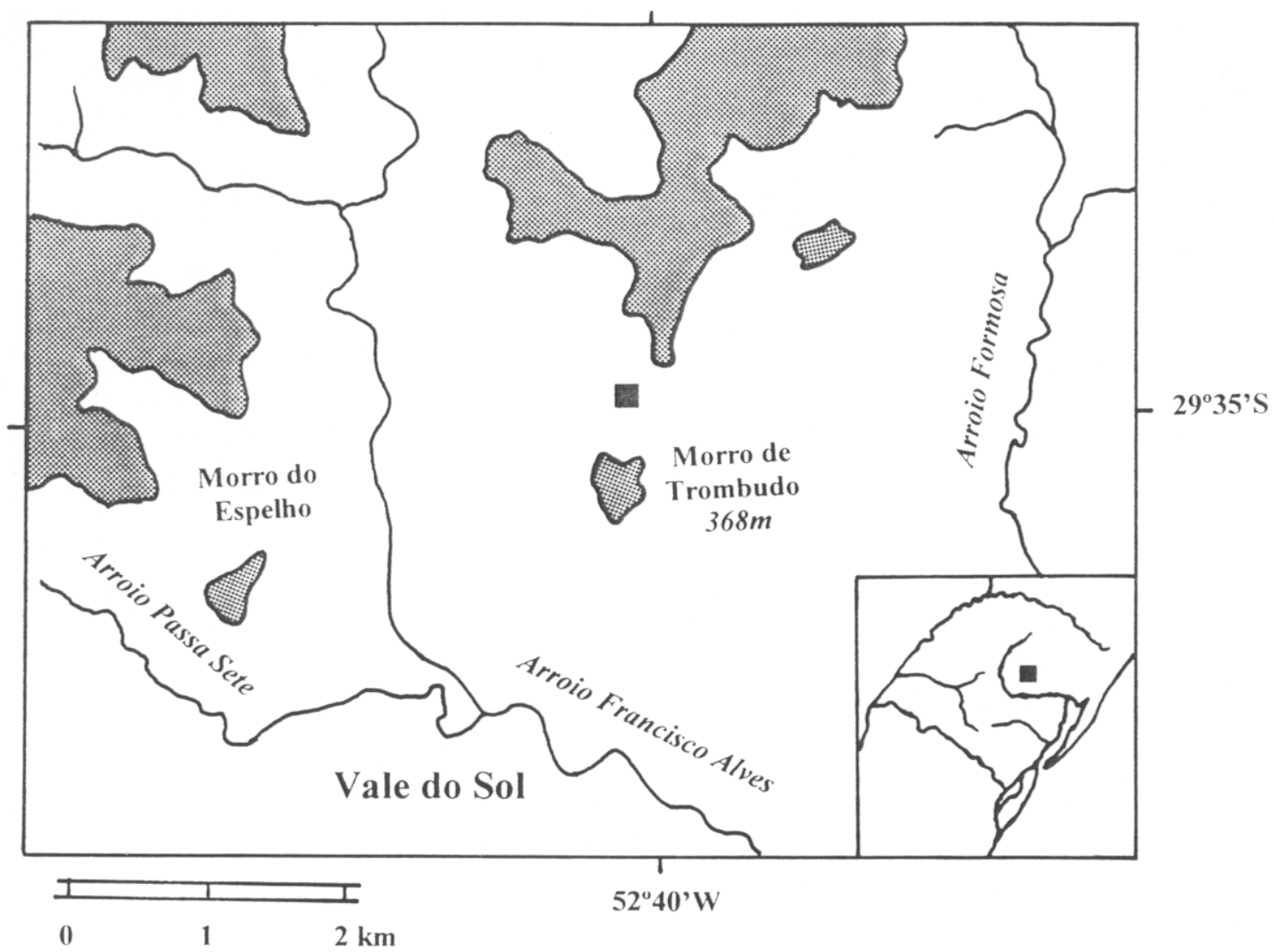

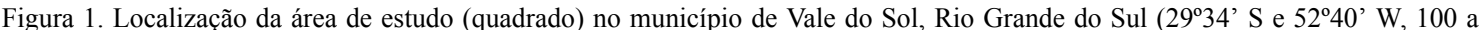
$140 \mathrm{msm}$ ). As áreas escurecidas correspondem a altitudes superiores a 300 m. Fonte: Ministério do Exército, Diretoria do Serviço Geográfico, Folha SH.22-H-III-2.

em um intervalo de 30 anos (IPAGRO 1979, 1989), mostram chuvas bem distribuídas durante o ano, com média anual de $1367 \mathrm{~mm}$, mas com dois períodos onde ocorrem médias inferiores a $100 \mathrm{~mm}$ (abril/maio e novembro), porém sem deficiência hídrica (Mota et al. 1970). A temperatura média anual, no período considerado, foi de $19,1^{\circ} \mathrm{C}$. A máxima registrada foi de $41,2{ }^{\circ} \mathrm{C}$ e a mínima de $-3,8{ }^{\circ} \mathrm{C}$, com uma média de 12 geadas anuais.

A área estudada situa-se em uma região que apresenta uma associação complexa de solos, com predomínio de Litólicos eutróficos, Cambissolo eutrófico, Brunizem Avermelhado e Terra Roxa Estruturada eutrófica (Ker et al. 1986).

A interpretação das análises químicas e granulométricas do solo, obtido a partir de 15 perfurações com um trado calador (tabela 1), baseada em critérios de fertilidade (Siqueira et al. 1987), revelaram um $\mathrm{pH}$ alto (em água) e teor médio de matéria orgânica. Os níveis de cálcio e magnésio também foram altos e o fósforo, em função do teor de argila, foi considerado baixo; o nível de potássio foi considerado suficiente. A saturação de bases (V) elevada confere ao solo boa fertilidade. As análises físicas demonstraram classe textural de argilosa (da superfície até $40 \mathrm{~cm}$ ) a média (de 40 a $60 \mathrm{~cm})$.
A amostragem fitossociológica foi realizada em 100 unidades amostrais contíguas de 10 por $10 \mathrm{~m}$ (1 ha). Todas as árvores vivas com diâmetro do caule a $1,3 \mathrm{~m}$ de altura do solo (DAP) a partir de $5 \mathrm{~cm}$ e altura mínima de $5 \mathrm{~m}$ foram amostradas (as árvores mortas e ainda em pé foram registradas à parte). Aquelas localizadas sobre as bordas das unidades amostrais foram consideradas, desde que no mínimo metade de seus diâmetros estivessem no interior da unidade amostral. De cada indivíduo amostrado foram tomadas as medidas de DAP e altura total. Exsicatas do material botânico coletado na área encontram-se nos acervos dos herbários dos Departamentos de Botânica da Universidade Federal de Pelotas (PEL) e da Universidade Federal do Rio Grande do Sul (ICN).

Os parâmetros fitossociológicos estimados foram: densidade, freqüência e dominância absolutas e valor de importância (Mueller-Dombois \& Ellenberg 1974). Na apresentação dos resultados, o valor de importância foi dividido por três.

Para a determinação da diversidade específica, utilizouse o índice H' de Shannon e, a equabilidade, foi estimada pelo índice J' de Pielou (Kent \& Coker 1992).

A avaliação da participação dos contingentes migratórios deu-se pela separação das espécies arbóreas, com base nos corredores seguidos para chegarem ao Rio Grande do Sul: se 
através do corredor atlântico ou leste, ou se ao longo das florestas em áreas aproximadamente coincidentes com as bacias dos rios Paraná e Uruguai, isto é, pelo oeste, excetuandose os pinhais e seus elementos típicos. As espécies amostradas que possuem ampla distribuição, foram tratadas como uma categoria distinta. Para o enquadramento das espécies nestas categorias, foram consultados diversos trabalhos sobre flora e/ou vegetação do sul do Brasil (Klein 1961, 1972, 1983, Rambo 1961, Reitz et al. 1983).

\section{Resultados}

As espécies amostradas foram 55, distribuídas em 46 gêneros de 23 famílias (tabela 2). Nas proximidades da área de amostragem, foram constatadas adicionalmente as presenças de Syagrus romanzoffiana (Arecaceae), Cedrela fissilis

Tabela 1. Características químicas e granulométricas* da amostra de solo coletada na área do levantamento fitossociológico em Vale do Sol (RS). Resultados expressos em material seco a $75{ }^{\circ} \mathrm{C}$.

\begin{tabular}{|c|c|c|c|}
\hline \multirow[b]{2}{*}{ Análises } & \multicolumn{3}{|c|}{ Profundidade $(\mathrm{cm})$} \\
\hline & $0-20$ & $20-40$ & $40-60$ \\
\hline pH (água) & 6,1 & 6,2 & 6,2 \\
\hline C orgânico (\%) & 4,3 & 2,3 & 1,8 \\
\hline $\mathrm{N}$ total $(\%)$ & 0,410 & 0,228 & 0,186 \\
\hline $\mathrm{P}$ assimilável $\left(\mu \mathrm{g} \cdot \mathrm{ml}^{-1}\right)$ & 6,0 & 4,0 & 5,0 \\
\hline $\mathrm{Ca}^{++}$trocável (mEq.100g $\left.\mathrm{g}^{-1}\right)$ & 21,3 & 17,8 & 17,5 \\
\hline $\mathrm{Mg}^{++}$trocável (mEq.100g $\left.{ }^{-1}\right)$ & 2,8 & 3,1 & 3,5 \\
\hline $\mathrm{K}^{+}$trocável (mEq.100g $\left.\mathrm{g}^{-1}\right)$ & 0,82 & 0,66 & 0,50 \\
\hline $\mathrm{Na}^{++}$trocável (mEq.100g $\left.\mathrm{g}^{-1}\right)$ & 0,14 & 0,13 & 0,16 \\
\hline $\mathrm{Al}^{+++}$trocável $\left(\mathrm{mEq} \cdot 100 \mathrm{~g}^{-1}\right)$ & 0,2 & 0,2 & 0,2 \\
\hline $\mathrm{H}^{+}$trocável (mEq.100g $\left.\mathrm{g}^{-1}\right)$ & 1,8 & 1,7 & 1,6 \\
\hline $\mathrm{H}+\mathrm{Al}\left(\mathrm{mEq} \cdot 100 \mathrm{~g}^{-1}\right)$ & 2,0 & 1,9 & 1,8 \\
\hline Soma de bases - S (mEq.100 $\left.{ }^{-1}\right)$ & 25,1 & 21,7 & 21,7 \\
\hline CTC - T (mEq.100g $\left.{ }^{-1}\right)$ & 27,1 & 23,6 & 23,5 \\
\hline Saturação de bases - V (\%) & 92,6 & 91,9 & 92,3 \\
\hline Saturação de Al - M (\%) & 0,74 & 0,85 & 0,85 \\
\hline Areia grossa $(\%)$ & 15,0 & 18,0 & 25,0 \\
\hline Areia fina (\%) & 11,0 & 13,0 & 15,0 \\
\hline Argila (\%) & 35,0 & 31,0 & 30,0 \\
\hline Silte (\%) & 39,0 & 38,0 & 30,0 \\
\hline Classe textural & argilosa & argilosa & média \\
\hline
\end{tabular}

*As análises foram realizadas segundo Tedesco et al. (1995), pelo Laboratório de Solos da Faculdade de Agronomia da Universidade Federal do Rio Grande do Sul.
(Meliaceae), Parapiptadenia rigida (Fabaceae), Ficus organensis (Moraceae) e Luehea divaricata (Tiliaceae). As famílias com maior riqueza específica foram Fabaceae e Myrtaceae, respectivamente com sete $(12,7 \%)$ e seis $(10,9 \%)$ espécies. Outras famílias relativamente ricas foram Euphorbiaceae, Lauraceae e Meliaceae com cinco espécies, Moraceae com quatro e outras seis famílias com duas, e as 11 restantes com uma única espécie.

Foram amostrados 1855 indivíduos no hectare, mais 35 árvores mortas em pé $(1,9 \%$ em relação às vivas). As três espécies com os maiores números de indivíduos foram Gymnanthes concolor, Euterpe edulis e Sorocea bonplandii (tabela 3), apresentando-se como típicas do sub-bosque, com indivíduos de pequena área basal. Outras, em função dos elevados diâmetros de seus troncos, apresentaram grandes valores de cobertura, como Erythrina falcata e Phytolacca dioica, que com pequeno número de indivíduos, inseriram-se entre as dez primeiras espécies em valor de importância.

Pachystroma longifolium e Nectandra megapotamica apareceram com um número intermediário de indivíduos, mas com os maiores valores de dominância.

Gymnanthes concolor e Euterpe edulis acumularam um terço do valor de importância, e somadas às sete espécies seguintes, acumularam $72,1 \%$ do total do valor de importância. As espécies com um único indivíduo amostrado, equivaleram a $20 \%$ do total. No entanto, a maioria destas espécies apresentou indivíduos de grande porte, sobretudo Patagonula americana e Myrocarpus frondosus.

A estimativa da diversidade arbórea pelo índice $H^{\prime}$ de Shannon resultou em 2,244 (nats) e a equabilidade J' de Pielou em 0,560. Ampliando o critério de inclusão para árvores com DAP a partir de $10 \mathrm{~cm}$, a riqueza sofreu apenas um pequeno decréscimo, apesar da expressiva redução no número de indivíduos. Para este critério, o índice de Shannon foi de 2,633, e a equabilidade de 0,666 (tabela 4).

A distribuição dos indivíduos segundo estimativas de altura (figura 2) permite reconhecer três grupos de árvores: o primeiro, entre seis e oito metros, apresenta um expressivo número de indivíduos; o segundo, entre nove e $19 \mathrm{~m}$, formando uma seqüência de alturas com número decrescente de indivíduos; o terceiro, acima de $20 \mathrm{~m}$, com um número muito reduzido de indivíduos. Esses grupos podem representar três estratos arbóreos na floresta, 
Tabela 2. Espécies, famílias e número de indivíduos, com o número de coleta de material testemunho, ocorrentes no levantamento realizado em Vale do Sol, RS, distribuídos nos respectivos corredores de imigração seguidos para chegarem ao Rio Grande do Sul ou como espécies de distribuição ampla. Números de coleta com J são do primeiro autor e com W do segundo; $\mathrm{NC}$ - não coletadas.

\begin{tabular}{|c|c|c|c|c|}
\hline Contingente & Espécie & Família & $\mathrm{N}^{\circ}$ indivíduos & $\mathrm{N}^{\circ}$ de coleta \\
\hline \multirow[t]{25}{*}{ Oeste } & Apuleia leiocarpa (Vogel) J.F. Macbr. & Fabaceae & 6 & $\mathrm{~J} 2288$ \\
\hline & Banara tomentosa Clos & Flacourtiaceae & 1 & J 2153 \\
\hline & Chrysophyllum gonocarpum (Mart. \& Eichler) Engler & Sapotaceae & 8 & J 2214 \\
\hline & Chrysophyllum marginatum (Hook. \& Arn.) Radlk. & Sapotaceae & 5 & J 3905 \\
\hline & Erythrina falcata Benth. & Fabaceae & 9 & W 585 \\
\hline & Eugenia involucrata DC. & Myrtaceae & 2 & $\mathrm{~J} 2154$ \\
\hline & Eugenia ramboi $\mathrm{D}$. Legrand & Myrtaceae & 2 & $\mathrm{~J} 2235$ \\
\hline & Eugenia rostrifolia D. Legrand & Myrtaceae & 34 & $\mathrm{~J} 2287$ \\
\hline & Hennecartia omphalandra Poiss. & Monimiaceae & 87 & $\mathrm{~J} 2140$ \\
\hline & Jacaranda micrantha Cham. & Bignoniaceae & 1 & J 2289 \\
\hline & Lonchocarpus campestris Mart. ex Benth. & Fabaceae & 2 & W 2542 \\
\hline & Lonchocarpus nitidus (Vogel) Benth. & Fabaceae & 2 & J 3910 \\
\hline & Machaerium paraguariense Hassl. & Fabaceae & 1 & W 1180 \\
\hline & Maclura tinctoria (L.) Don ex Steud. & Moraceae & 4 & W 1525 \\
\hline & Myrcianthes pungens (O. Berg) D. Legrand & Myrtaceae & 3 & W 1730 \\
\hline & Myrocarpus frondosus $\mathrm{M}$. Allemão & Fabaceae & 1 & W 1779 \\
\hline & Myrsine loefgrenii (Mez) Otegui & Myrsinaceae & 3 & J 2245 \\
\hline & Ocotea diospyrifolia (Meisn.) Mez & Lauraceae & 4 & $\mathrm{~J} 2223$ \\
\hline & Patagonula americana $\mathrm{L}$. & Boraginaceae & 1 & W 1785 \\
\hline & Pilocarpus pennatifolius Lem. & Rutaceae & 4 & J 2130 \\
\hline & Ruprechtia laxiflora Meisn. & Polygonaceae & 1 & $\mathrm{~J} 2222$ \\
\hline & Schefflera morototoni (Aubl.) Maguire, Steyermark \& Frodin & Araliaceae & 3 & $\mathrm{~J} 2351$ \\
\hline & Trichilia claussenii C. DC. & Meliaceae & 71 & $\mathrm{~J} 2221$ \\
\hline & Trichilia elegans A. Juss. & Meliaceae & 8 & $\mathrm{~J} 2215$ \\
\hline & Urera baccifera (L.) Gaudich. & Urticaceae & 14 & $\mathrm{~J} 2211$ \\
\hline \multirow[t]{10}{*}{ Leste } & Aiouea saligna Meisn. & Lauraceae & 21 & J 2292 \\
\hline & Eugenia schuechiana O. Berg & Myrtaceae & 2 & J 3904 \\
\hline & Euterpe edulis Mart. & Arecaceae & 434 & J 2290 \\
\hline & Faramea marginata Cham. & Rubiaceae & 16 & J 2286 \\
\hline & Ficus insipida Willd. & Moraceae & 3 & W 1194 \\
\hline & Margaritaria nobilis L. f. & Euphorbiaceae & 1 & J 2169 \\
\hline & Mollinedia schottiana (Spreng.) Perkins & Monimiaceae & 8 & J 1811 \\
\hline & Ocotea silvestris Vattimo & Lauraceae & 2 & J 2293 \\
\hline & Pachystroma longifolium (Nees) I.M. Johnst. & Euphorbiaceae & 46 & W 1479 \\
\hline & Trichilia pallens $\mathrm{C} . \mathrm{DC}$. & Meliaceae & 21 & J 2246 \\
\hline \multirow[t]{20}{*}{ Amplo } & Alchornea triplinervia (Spreng.) Müll. Arg. & Euphorbiaceae & 7 & J 1936 \\
\hline & Allophylus edulis (A. St.-Hil.) Radlk. & Sapindaceae & 4 & $\mathrm{~J} 2133$ \\
\hline & Boehmeria caudata $\mathrm{Sw}$. & Urticaceae & 5 & $\mathrm{~J} 2160$ \\
\hline & Cabralea canjerana (Vell.) Mart. & Meliaceae & 9 & $\mathrm{NC}$ \\
\hline & Campomanesia xanthocarpa $\mathrm{O}$. Berg & Myrtaceae & 2 & W 588 \\
\hline & Casearia silvestris $\mathrm{Sw}$. & Flacourtiaceae & 1 & W 584 \\
\hline & Cordia trichotoma (Vell.) Arráb. ex Steud. & Boraginaceae & 1 & $\mathrm{NC}$ \\
\hline & Cupania vernalis Cambess. & Sapindaceae & 9 & $\mathrm{NC}$ \\
\hline & Endlicheria paniculata (Spreng.) J.F. Macbr. & Lauraceae & 2 & $\mathrm{~J} 2127$ \\
\hline & Ficus luschnathiana (Miq.) Miq. & Moraceae & 1 & W 1476 \\
\hline & Guarea macrophylla Vahl & Meliaceae & 3 & J 2220 \\
\hline & Gymnanthes concolor Spreng. & Euphorbiaceae & 632 & $\mathrm{NC}$ \\
\hline & Inga semialata (Vell.) Mart. & Fabaceae & 36 & $\mathrm{~J} 2280$ \\
\hline & Nectandra megapotamica (Spreng.) Mez & Lauraceae & 21 & J 2129 \\
\hline & Pisonia ambigua Heimerl & Nyctaginaceae & 3 & $\mathrm{NC}$ \\
\hline & Phytolacca dioica $\mathrm{L}$. & Phytolaccaceae & 6 & W 583 \\
\hline & Solanum sanctaecatharinae Dunal & Solanaceae & 1 & J 2213 \\
\hline & Sorocea bonplandii (Baill.) Burger, Lanj. \& Boer & Moraceae & 255 & $\mathrm{~J} 2155$ \\
\hline & Tetrorchidium rubrivenium Poepp. \& Endl. & Euphorbiaceae & 20 & W 2082 \\
\hline & Trema micrantha (L.) Blume & Ulmaceae & 6 & $\mathrm{~J} 2161$ \\
\hline
\end{tabular}


Tabela 3. Parâmetros fitossociológicos estimados para as espécies arbóreas amostradas em Vale do Sol (RS), em ordem decrescente de valores de importância (VI). DA - densidade absoluta; FA - freqüência absoluta; CA - cobertura caulinar ou dominância absoluta.

\begin{tabular}{|c|c|c|c|c|c|}
\hline & Espécie & DA (ind.ha ${ }^{-1}$ ) & FA (\%) & $\mathrm{CA}\left(\mathrm{m}^{2} \cdot \mathrm{ha}^{-1}\right)$ & VI (\%) \\
\hline 1. & Gymnanthes concolor & 632,0 & 99,0 & 3,439 & 18,81 \\
\hline 2. & Euterpe edulis & 434,0 & 88,0 & 3,480 & 14,77 \\
\hline 3. & Sorocea bonplandii & 255,0 & 88,0 & 1,158 & 9,69 \\
\hline 4. & Pachystroma longifolium & 46,0 & 35,0 & 4,374 & 5,99 \\
\hline 5. & Trichilia claussenii & 71,0 & 49,0 & 2,928 & 5,95 \\
\hline 6. & Hennecartia omphalandra & 87,0 & 57,0 & 2,056 & 5,92 \\
\hline 7. & Nectandra megapotamica & 21,0 & 19,0 & 3,784 & 4,31 \\
\hline 8. & Erythrina falcata & 9,0 & 9,0 & 3,731 & 3,58 \\
\hline 9. & Phytolacca dioica & 6,0 & 6,0 & 3,372 & 3,09 \\
\hline 10. & Eugenia rostrifolia & 34,0 & 26,0 & 1,097 & 2,72 \\
\hline 11. & Inga semialata & 36,0 & 25,0 & 0,484 & 2,22 \\
\hline 12. & Tetrorchidium rubrivenium & 20,0 & 18,0 & 1,162 & 2,14 \\
\hline 13. & Trichilia pallens & 21,0 & 18,0 & 0,415 & 1,56 \\
\hline 14. & Aiouea saligna & 21,0 & 19,0 & 0,305 & 1,52 \\
\hline 15. & Cupania vernalis & 9,0 & 9,0 & 1,099 & 1,47 \\
\hline 16. & Alchornea triplinervia & 7,0 & 7,0 & 1,261 & 1,47 \\
\hline 17. & Cabralea canjerana & 9,0 & 8,0 & 0,950 & 1,30 \\
\hline 18. & Faramea marginata & 16,0 & 15,0 & 0,076 & 1,06 \\
\hline 19. & Apuleia leiocarpa & 6,0 & 6,0 & 0,694 & 0,95 \\
\hline 20. & Urera baccifera & 14,0 & 11,0 & 0,189 & 0,92 \\
\hline 21. & Ocotea diospyrifolia & 4,0 & 4,0 & 0,708 & 0,83 \\
\hline 22. & Chrysophyllum gonocarpum & 8,0 & 7,0 & 0,199 & 0,64 \\
\hline 23. & Maclura tinctoria & 4,0 & 4,0 & 0,421 & 0,60 \\
\hline 24. & Chrysophyllum marginatum & 5,0 & 5,0 & 0,333 & 0,59 \\
\hline 25. & Trichilia elegans & 8,0 & 8,0 & 0,045 & 0,56 \\
\hline 26. & Mollinedia schottiana & 8,0 & 8,0 & 0,026 & 0,54 \\
\hline 27. & Myrcianthes pungens & 3,0 & 2,0 & 0,483 & 0,53 \\
\hline 28. & Allophylus edulis & 4,0 & 4,0 & 0,323 & 0,52 \\
\hline 29. & Schefflera morototoni & 3,0 & 3,0 & 0,397 & 0,51 \\
\hline 30. & Lonchocarpus campestris & 2,0 & 2,0 & 0,333 & 0,40 \\
\hline 31. & Ficus insipida & 3,0 & 3,0 & 0,167 & 0,33 \\
\hline 32. & Patagonula americana & 1,0 & 1,0 & 0,303 & 0,31 \\
\hline 33. & Trema micrantha & 6,0 & 3,0 & 0,056 & 0,29 \\
\hline 34. & Ocotea silvestris & 2,0 & 2,0 & 0,171 & 0,27 \\
\hline 35. & Pisonia ambigua & 3,0 & 2,0 & 0,137 & 0,26 \\
\hline 36. & Campomanesia xanthocarpa & 2,0 & 2,0 & 0,159 & 0,26 \\
\hline 37. & Pilocarpus pennatifolius & 4,0 & 3,0 & 0,029 & 0,24 \\
\hline 38. & Boehmeria caudata & 5,0 & 2,0 & 0,033 & 0,21 \\
\hline 39. & Myrocarpus frondosus & 1,0 & 1,0 & 0,177 & 0,20 \\
\hline 40. & Guarea macrophylla & 3,0 & 3,0 & 0,008 & 0,20 \\
\hline 41. & Jacaranda micrantha & 1,0 & 1,0 & 0,162 & 0,19 \\
\hline 42. & Eugenia ramboi & 2,0 & 2,0 & 0,077 & 0,19 \\
\hline 43. & Margaritaria nobilis & 1,0 & 1,0 & 0,156 & 0,19 \\
\hline 44. & Lonchocarpus nitidus & 2,0 & 2,0 & 0,064 & 0,18 \\
\hline 45. & Ruprechtia laxiflora & 1,0 & 1,0 & 0,137 & 0,17 \\
\hline 46. & Myrsine loefgrenii & 3,0 & 2,0 & 0,025 & 0,17 \\
\hline 47. & Eugenia involucrata & 2,0 & 2,0 & 0,041 & 0,16 \\
\hline 48. & Machaerium paraguariense & 1,0 & 1,0 & 0,117 & 0,16 \\
\hline 49. & Cordia trichotoma & 1,0 & 1,0 & 0,117 & 0,16 \\
\hline 50. & Eugenia schuechiana & 2,0 & 2,0 & 0,015 & 0,14 \\
\hline 51. & Endlicheria paniculata & 2,0 & 2,0 & 0,010 & 0,14 \\
\hline 52. & Banara tomentosa & 1,0 & 1,0 & 0,078 & 0,13 \\
\hline 53. & Solanum sanctaecatharinae & 1,0 & 1,0 & 0,028 & 0,09 \\
\hline 54. & Ficus luschnathiana & 1,0 & 1,0 & 0,020 & 0,08 \\
\hline 55. & Casearia silvestris & 1,0 & 1,0 & 0,013 & 0,07 \\
\hline
\end{tabular}


Tabela 4. Dados de alguns levantamentos realizados no Rio Grande do Sul, com indicação dos respectivos métodos de amostragem, diâmetro mínimo de inclusão (DAP), número de espécies amostradas (Ne), número de indivíduos amostrados (Ni) e o índice de diversidade de Shannon (H').

\begin{tabular}{llcccc}
\hline Levantamentos & Método & $\mathrm{DAP}$ & $\mathrm{Ne}$ & $\mathrm{Ni}$ & $\mathrm{H}^{\prime}$ \\
\hline Este estudo & Parcelas (1 ha) & $5 \mathrm{~cm}$ & 55 & 1855 & 2,244 \\
Jarenkow \& Baptista (1987) & Parcelas (0,48 ha) & $5 \mathrm{~cm}$ & 38 & 353 & 2,93 \\
Dillenburg et al. (1992) & Quadrantes (45 pontos) & $5 \mathrm{~cm}$ & 15 & 180 & 1,975 \\
Este estudo & Parcelas (1 ha) & $10 \mathrm{~cm}$ & 52 & 782 & 2,633 \\
Vasconcellos et al. (1992) & Quadrantes (107 pontos) & $10 \mathrm{~cm}$ & 61 & 428 & 3,52 \\
Bencke \& Soares (1998) & Parcelas (0,3 ha) & $10 \mathrm{~cm}$ & 44 & 214 & 3,22 \\
Waechter \& Jarenkow (1998) & Quadrantes (30 pontos) & $10 \mathrm{~cm}$ & 12 & 120 & 1,886 \\
\hline
\end{tabular}

um inferior, um médio e um superior. As maiores medidas individuais de altura e diâmetro foram de $27 \mathrm{~m}$ (Erythrina falcata) e $119 \mathrm{~cm}$ (Phytolacca dioica), respectivamente.

A avaliação da influência dos contingentes migratórios na composição qualitativa (tabela 5), revelou maior número de espécies provindas do oeste, isto é, das espécies paranaense-uruguaias (45,5\%). Seguiram as espécies de ampla distribuição $(36,4 \%)$ e, por último, as espécies do contingente leste ou atlântico $(18,2 \%)$. Considerando a participação quantitativa, as espécies de ampla distribuição superaram nitidamente $(55,2 \%)$ os contingentes leste $(29,9 \%)$ e oeste $(14,9 \%)$. Apesar do grande número de indivíduos das espécies do contingente leste, a dominância relativa foi bem menor em relação às do oeste, principalmente pela alta densidade e reduzidos diâmetros de Euterpe edulis.

\section{Discussão}

As espécies presentes na área de estudo são citadas em diversos estudos anteriores realizados na região central do Rio Grande do Sul (Klein 1983, 1984b, Longhi et al. 1986, Farias et al. 1994, Bencke \& Soares 1998), indicando pouca variação florística regional. A predominância de leguminosas coincide com outros levantamentos efetuados em florestas estacionais ocorrentes ao longo das bacias dos rios ParanáUruguai (Martins 1991, Vasconcellos et al. 1992, Stutz-de-Ortega 1987). Nas florestas ombrófilas, atlântica e com araucária, a participação de leguminosas é bem menor (Mori et al. 1983, Jarenkow \& Baptista 1987). A vantagem sobre espécies de outras famílias possivelmente está relacionada à capacidade de obter nitrogênio a partir de relações simbióticas com bactérias (Cavassan et al. 1984, Martins 1991).
A comparação da densidade total por área obtida com outros trabalhos realizados no sul do Brasil, torna-se difícil diante das diferenças metodológicas empregadas (Veloso \& Klein 1961, 1968, Bencke \& Soares 1998, Waechter \& Jarenkow 1998). Em florestas no Rio Grande do Sul, considerando o mesmo critério de inclusão (DAP $\geq 5 \mathrm{~cm}$ ), as estimativas da densidade arbórea total (tabela 4) variam de 735 indivíduos.ha ${ }^{-1}$, em uma floresta com araucária (Jarenkow \& Baptista 1987), até 2219 indivíduos.ha $^{-1}$, em uma floresta de restinga de pequeno porte (Dillenburg et al. 1992). Esses números sugerem um gradiente de densidade decrescente a partir de matas de planície para matas de encosta e planalto, possivelmente relacionado com o porte dos indivíduos.

As três espécies com maior número de indivíduos, Gymnanthes concolor, Euterpe edulis e Sorocea bonplandii, são típicas do sub-bosque de vários tipos florestais no sul do Brasil (Klein 1961, 1983, Reitz et al. 1983). Em levantamentos realizados no estado, G. concolor assim como S. bonplandii, quando amostradas, apareceram com baixas densidades, exceto no Parque Estadual do Turvo (município de Derrubadas), onde esta última espécie contribuiu com o maior valor de importância (Vasconcellos et al. 1992). O grande número de indivíduos amostrados de Euterpe edulis demonstra a densidade variável que a espécie apresenta ao longo de matas na encosta meridional no Rio Grande do Sul, já que em alguns levantamentos, poucos ou nenhum indivíduo foram amostrados (Longhi et al. 1986, Daniel 1991, Machado \& Longhi 1991). E. edulis mostra-se igualmente abundante em diversos levantamentos realizados em outros locais da Região Sul, apesar da intensa exploração a que está submetido (Veloso \& Klein 1957, 1961, 1963, 1968, Soares-Silva \& Barroso 1992).

O percentual de espécies com um indivíduo amostrado neste estudo está entre os mais baixos 


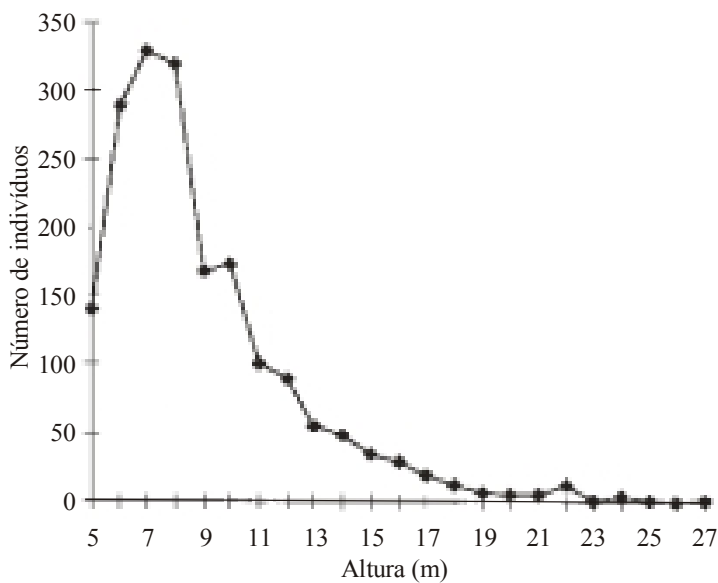

Figura 2. Distribuição das alturas dos indivíduos arbóreos amostrados em Vale do Sol (RS).

registrados em levantamentos florestais quantitativos realizados no estado, que variam entre 19,5\% (Vasconcellos et al. 1992) e 47,7\% (Bencke \& Soares 1998). De modo geral, verifica-se que a composição de espécies com um índivíduo amostrado nesses levantamentos é bastante diferente.

O índice de diversidade, visto ao lado de outros valores encontrados para florestas no estado (tabela 4), pode ser considerado intermediário entre os baixos valores constatados em florestas de restinga (Dillenburg et al. 1992, Waechter \& Jarenkow 1998), bastante pobres floristicamente, e os valores mais altos encontrados em florestas com araucária (Jarenkow \& Baptista 1987), florestas estacionais (Machado \& Longhi 1991, Vasconcellos et al. 1992, Bencke \& Soares 1998) e florestas ombrófilas costeiras (Jarenkow 1994).

A menor diversidade estimada para Vale do Sol, em relação a outras florestas estacionais, pode, em parte, ser explicada pela baixa equabilidade encontrada, resultante do elevado número de indivíduos de algumas espécies amostradas, principalmente Gymnanthes concolor, Euterpe edulis e Sorocea bonplandii. Mesmo considerando árvores com DAP a partir de $10 \mathrm{~cm}$, a diversidade específica continua relativamente baixa em relação as outras florestas estacionais, indicando uma possível influência do tamanho amostral.

Os estratos reconhecidos neste trabalho discordam daqueles definidos por Klein (1983), para florestas estacionais da Serra Geral. Estas diferenças provavelmente devem-se às estimativas mais
Tabela 5. Número de espécies e de indivíduos e a soma dos parâmetros fitossociológicos das espécies amostradas em Vale do Sol (RS), pelos respectivos corredores de migração seguidos para chegarem ao Estado (leste ou oeste) ou como espécies de ampla distribuição.

\begin{tabular}{lrrr}
\hline Parâmetros fitossociológicos & Leste & Oeste & Ampla \\
\hline Número de espécies & 10,0 & 25,0 & 20,0 \\
Número de indivíduos & 554,0 & 277,0 & 1024,0 \\
Densidade relativa & 29,9 & 14,9 & 55,2 \\
Freqüência relativa & 27,2 & 29,9 & 42,9 \\
Dominância relativa & 22,1 & 35,6 & 42,3 \\
Valor de importância & 26,4 & 25,8 & 46,8 \\
\hline
\end{tabular}

detalhadas no presente estudo, ou seja, para cada indivíduo amostrado, e a uma possível exploração seletiva de árvores de grande porte, já que não foram observados indivíduos emergentes entre 30 e $35 \mathrm{~m}$ de altura.

Quanto à participação de espécies dos contingentes migratórios na composição florística da área estudada, a predominância daquelas provindas da rota oeste coincide com as observações de Rambo (1961), que destaca a importância deste contingente nas florestas interiores do Rio Grande do Sul. Hennecartia omphalandra, Trichilia claussenii e Eugenia rostrifolia são as espécies mais comuns deste contingente. Algumas espécies que ocorreram com poucos indivíduos, como Erythrina falcata, Apuleia leiocarpa, Maclura tinctoria, Schefflera morototoni e Cordia trichotoma, são fisionomicamente conspícuas, pelo grande porte que atingem na floresta.

Os parâmetros mais altos para o contingente de ampla distribuição provavelmente refletem a importância de espécies de grande amplitude ecológica na composição das florestas estacionais do sul do Brasil, que estão presentes com densidades variáveis em distintas fases sucessionais. Gymnanthes concolor e Sorocea bonplandii contribuem com a maioria dos indivíduos deste grupo. Outras espécies ainda representativas são Inga semialata, Nectandra megapotamica e Tetrorchidium rubrivenium.

As espécies do contingente leste ou atlântico têm em Euterpe edulis o seu mais numeroso e característico representante. Na região subtropical da América do Sul a espécie ocorre no leste do Paraguai, no nordeste da Argentina e no sul do Brasil, onde é encontrada no oeste paranaense, contudo sem alcançar a região do Alto Uruguai, tanto no lado catarinense quanto no sul-rio-grandense (Reitz 1974, 
Rambo 1961). É pelo corredor atlântico que chega ao Rio Grande do Sul, avançando até a porção norte da mata da encosta da Serra do Sudeste e, ao longo da encosta meridional do Planalto Sul-Brasileiro, até a região central do estado (próximo a Candelária). Entre as espécies atlânticas, ainda se destacam Pachystroma longifolium, Trichilia pallens e Aiouea saligna.

O principal aspecto conclusivo do presente trabalho, que deriva da abordagem fitossociológica e fitogeográfica, revela que as espécies do contingente leste contribuem com um grande número de indivíduos, mas predominam no sub-bosque como árvores de tamanho médio. $\mathrm{O}$ contingente oeste, por sua vez, apresenta maior riqueza, mas contribui com um menor número de indivíduos que são fisionomicamente mais importantes, muitas vezes emergentes, aparentemente formando condições favoráveis para o desenvolvimento de algumas espécies atlânticas tolerantes à sombra.

Agradecimentos - Os autores agradecem a Marcos Sobral, pelo auxílio na identificação de material botânico e ao Sr. Adelário Schreder e Família Prantl, por possibilitarem o acesso às áreas de estudo.

\section{Referências Bibliográficas}

BAPTISTA, L.R.M. 1967. Sobre uma comunidade florestal em Morungava (Município de Gravataí, RS). In Anais do XV Congresso Brasileiro de Botânica, Porto Alegre, p.197-201.

BAPTISTA, L.R.M. 1976. A floresta tropical no Rio Grande do Sul. In Anais do II Congresso Brasileiro de Florestas Tropicais, Mossoró, p.125-128.

BENCKE, C.S.C. \& SOARES, C. 1998. Estudo fitossociológico da vegetação arbórea de uma área de floresta estacional em Santa Cruz do Sul, RS, Brasil. Caderno de Pesquisa, Série Botânica 10:37-57.

CAVASSAN, O., CESAR, O. \& MARTINS, F.R. 1984. Fitossociologia da vegetação arbórea da Reserva Estadual de Bauru, estado de São Paulo. Revista Brasileira de Botânica 7:91-106.

DANIEL, A. 1991. Estudo fitossociológico arbóreo/arbustivo da mata ripária da bacia hidrográfica do Rio dos Sinos, RS. Pesquisas, Série Botânica 41:5-199.

DILLENBURG, L.R., WAECHTER, J.L. \& PORTO, M.L. 1992. Species composition and structure of a sandy coastal plain forest in northern Rio Grande do Sul, Brazil. In Coastal plant communities of Latin America (U. Seeliger, ed.). Academic Press, San Diego, p.349-366.

FARIAS, J.A.C., TEIXEIRA, I.F., PES, S. \& ALVAREZFILHO, A. 1994. Estrutura fitossociológica de uma floresta estacional decidual na região de Santa Maria, RS. Ciência Florestal 1:109-128.
IBDF (Instituto Brasileiro de Desenvolvimento Florestal). 1983. Inventário florestal nacional: florestas nativas, Rio Grande do Sul. Instituto Brasileiro de Desenvolvimento Florestal, Brasília.

IPAGRO (Instituto de Pesquisas Agropecuárias). 1979. Observações meteorológicas no estado do Rio Grande do Sul. Boletim Técnico 3:1-272.

IPAGRO (Instituto de Pesquisas Agropecuárias). 1989. Atlas agroclimático do estado do Rio Grande do Sul. Secretaria da Agricultura e Abastecimento, Porto Alegre, v.1.

JARENKOW, J.A. 1994. Estudo fitossociológico comparativo entre duas áreas com Mata de Encosta no Rio Grande do Sul. Tese de doutorado, Universidade Federal de São Carlos, São Carlos.

JARENKOW, J.A. \& BAPTISTA, L.R.M. 1987. Composição florística e estrutura da mata com araucária na Estação Ecológica de Aracuri, Esmeralda, Rio Grande do Sul. Napaea 3:9-18.

KAUL, P.F.T. 1990. Geologia. In Geografia do Brasil; Região Sul. Instituto Brasileiro de Geografia e Estatística, Rio de Janeiro, v. 2, p.29-54.

KENT, M. \& COKER, P. 1992. Vegetation description and analysis; a practical approach. Belhaven, London.

KER, J.C., ALMEIDA, J.A., FASOLO, P.J. \& HOCHMULLER, D.P. 1986. Pedologia - levantamento exploratório de solos. In Levantamento de recursos naturais. Instituto Brasileiro de Geografia e Estatística, Rio de Janeiro, v. 33, p.405-540.

KLEIN, R.M. 1961. Aspectos fitofisionômicos da mata pluvial da costa atlântica do sul do Brasil. Boletin de la Sociedad Argentina de Botánica 9:121-140.

KLEIN, R.M. 1972. Árvores nativas da Floresta Subtropical do Alto Uruguai. Sellowia 24:9-62.

KLEIN, R.M. 1983. Aspectos fitofisionômicos da floresta estacional na fralda da Serra Geral (RS). In Anais do XXXIV Congresso Nacional de Botânica, Porto Alegre, p.73-110.

KLEIN, R.M. 1984a. Aspectos dinâmicos da vegetação do sul do Brasil. Sellowia 36:5-54.

KLEIN, R.M. 1984b. Síntese ecológica da Floresta Estacional da Bacia do Jacuí e importância do reflorestamento com essências nativas (RS). In Anais do V Congresso Florestal Estadual, Nova Prata, p.265-278.

LEITE, P.F. \& KLEIN, R.M. 1990. Vegetação. In Geografia do Brasil: Região Sul. Instituto Brasileiro de Geografia e Estatística, Rio de Janeiro, v. 2, p.113-150.

LINDMAN, C.A.M. 1906. A vegetação no Rio Grande do Sul. Universal, Porto Alegre.

LONGHI, S.J., SANTOS, P. \& SCHORN, L.A. 1986. Diferenciação dos tipos florestais do Morro do Botucaraí, em Candelária, Rio Grande do Sul. Acta Florestal Brasileira 1:99-114.

MACHADO, P.F.S. \& LONGHI, S.J. 1991. Aspectos florísticos e fitossociológicos da floresta do Morro Osório, RS, Brasil. Ciência e Natura 13:103-116.

MARTINS, F.R. 1991. Estrutura de uma floresta mesófila. Editora da Universidade Estadual de Campinas, Campinas.

MORENO, J.A. 1961. Clima do Rio Grande do Sul. Secretaria da Agricultura do Rio Grande do Sul, Porto Alegre.

MORI, S.C., BOOM, B.M., CARVALHO, A.M. \& SANTOS, T.S. 1983. Ecological importance of Myrtaceae in a eastern Brazilian forest. Biotropica 15:68-70. 
MOTA, F.S., GOEDERT, C.O., LOPES, N.F., GARCEZ, J.R.B. \& GOMES, A.S. 1970. Balanço hídrico do Rio Grande do Sul. Pesquisa Agropecuária Brasileira 5:1-27.

MUELLER-DOMBOIS, D. \& ELLENBERG, H. 1974. Aims and methods of vegetation ecology. John Wiley, New York.

RAMBO, B. 1951. A imigração da selva higrófila no Rio Grande do Sul. Anais Botânicos do Herbário Barbosa Rodrigues 3:55-91.

RAMBO, B. 1956. A fisionomia do Rio Grande do Sul. 2ed. Selbach, Porto Alegre.

RAMBO, B. 1960. Die Südgrenze des brasilianischen Regenwaldes. Pesquisas, Botânica 8:5-41.

RAMBO, B. 1961. Migration routes of the South Brazilian rain forest. Pesquisas, Botânica 12:1-54.

REITZ, R. 1974. Palmeiras. In Flora ilustrada catarinense (R. Reitz, ed.). Herbário Barbosa Rodrigues, Itajaí, p.3-189.

REITZ, R., KLEIN, R.M. \& REIS, A. 1983. Projeto madeira do Rio Grande do Sul. Sellowia 34/35:1-525.

SCHULTZ, A.R. 1957. Some fitogeografical and fitological data from Rio Grande do Sul, Brazil. Vegetatio 7:355-360.

SIQUEIRA, O.J.F, SCHERER, E.E., TASSINARI, G., ANGHINON, I., PATELA, J.F., TEDESCO, J.F., MILAN, P.A. \& ERNANI, P.R. 1987. Recomendações de adubação e calagem para os estados do Rio Grande do Sul e Santa Catarina. EMBRAPA-CNPT, Passo Fundo.

SOARES-SILVA, L.H. \& BARROSO, G.M. 1992. Fitossociologia do estrato arbóreo da floresta na porção norte do Parque Estadual Mata dos Godoy, Londrina-PR, Brasil. In Anais do VIII Congresso da Sociedade Botânica de São Paulo, Campinas, p.101-112.

STUTZ-DE-ORTEGA, L.C. 1987. Etudes floristiques de divers stades secondaires des formations forestières du Haut Parana (Paraguay oriental). Structure, composition floristique et régénération naturelle: comparaison entre forêt primaire et la forêt selectivement exploite. Candollea 42:205-262.

TEDESCO, M.J., GIANELLO, C., BISSANI, C.A., BOHNEN, H. \& VOLKWEISS, S.J. 1995. Análise de solo, plantas e outros materiais. Universidade Federal do Rio Grande do Sul, Porto Alegre.
TEIXEIRA, M.B., COURA-NETO, A.B., PASTORE, U. \& RANGEL FILHO, A.L.R. 1986. Vegetação; as regiões fitoecológicas, sua natureza, seus recursos econômicos; estudo fitogeográfico. In Levantamento de recursos naturais. Instituto Brasileiro de Geografia e Estatística, Rio de Janeiro, v.33, p.541-632.

VASCONCELLOS, J.M.O., DIAS, L.L., SILVA, C.P. \& SOBRAL, M. 1992. Fitossociologia de uma área de mata subtropical no Parque Estadual do Turvo, RS. Revista do Instituto Florestal 4:252-259.

VELOSO, H.P. \& GÓES-FILHO, L. 1982. Fitogeografia brasileira - classificação fisionômico-ecológica da vegetação neotropical. Boletim Técnico do Projeto RADAMBRASIL, Série Vegetação 1:1-80.

VELOSO, H.P. \& KLEIN, R.M. 1957. As comunidades e associações vegetais da mata pluvial do sul do Brasil - I. As comunidades do município de Brusque, estado de Santa Catarina. Sellowia 9:81-235.

VELOSO, H.P. \& KLEIN, R.M. 1961. As comunidades e associações vegetais da mata pluvial do sul do Brasil: III As associações vegetais das planícies costeiras do quaternário, situadas entre o Rio Itapocu (Estado de Santa Catarina) e a Baia de Paranaguá (estado do Paraná). Sellowia 13:205-260.

VELOSO, H.P. \& KLEIN, R.M. 1963. As comunidades e associações vegetais da mata pluvial do sul do Brasil: IV. As associações situadas entre o Rio Tubarão e a Lagoa dos Barros. Sellowia 15:57-114.

VELOSO, H.P. \& KLEIN, R.M. 1968. As comunidades e associações vegetais da mata pluvial do sul do Brasil VI. Agrupamentos arbóreos dos contra-fortes da Serra Geral situados ao sul da costa catarinense e ao norte da costa sul-riograndense. Sellowia 20:127-180.

WAECHTER, J.L. 1998. Epiphytic orchids in eastern subtropical South America. In Proceedings of the $15^{\text {th }}$ World Orchid Conference, Turriers, p.332-341.

WAECHTER, J.L. \& JARENKOW, J.A. 1998. Composição e estrutura do componente arbóreo nas matas turfosas do Taim, Rio Grande do Sul. Biotemas 11:45-69. 